\title{
Respiratory System Deposition with a Novel Aerosol Delivery System in Spontaneously Breathing Healthy Adults
}

\author{
Allan L Coates MDCM, Kitty Leung, Jeffrey Chan, Nancy Ribeiro RTNM, \\ Martin Charron MD, and Suzanne Schuh MD
}

\begin{abstract}
BACKGROUND: Intravenous magnesium sulfate $\left(\mathrm{MgSO}_{4}\right)$ in children and adults with refractory acute asthma is effective, but therapy may be limited by systemic hypotension that might be avoided with the aerosol route. Inhaled $\mathrm{MgSO}_{4}$ has a relatively high dose (volume) requirement. This, plus the use of inefficient delivery systems, may explain the lack of efficacy of inhaled $\mathrm{MgSO}_{4}$ in some studies. An in vitro study suggested that the AeroNeb Go with the Idehaler Pocket and a face mask would deliver $16 \mathrm{mg} / \mathrm{min}$ of $\mathrm{MgSO}_{4}$ to the respiratory system in older children, and approximately a fifth for toddlers, but no in vivo data exist. METHODS: Saline mixed with a radiolabel was used as a proxy for the $100 \mathrm{mg} / \mathrm{mL} \mathrm{MgSO}_{4}$ solution. In 5 adult males the rate of deposition was measured using nuclear medicine techniques. The radiolabel deposition below the vocal cords was converted to the rate of deposition of $\mathrm{MgSO}_{4}$ and compared to the results from an in vitro model using adult respiratory patterns. RESULTS: The mean \pm SD rate of deposition was $12.6 \pm 1.9 \mathrm{mg} / \mathrm{min}$. The reasons for this lower deposition, compared to the in vitro estimate, was most likely the exhalation of anatomical dead space aerosol, which would have been captured on the inspiratory filter in vitro. CONCLUSIONS: These in vivo data confirm the deposition data predicted in the in vitro study, although caution should be used in extrapolating the results to children. This device appears suitable for the clinical trial of inhaled $\mathrm{MgSO}_{4}$ in children and adults with refractory asthma. Key words: asthma; inhaled magnesium sulfate; nuclear medicine; respiratory system deposition; pulmonary deposition. [Respir Care 2013;58(12):2087-2092. (c) 2013 Daedalus Enterprises]
\end{abstract}

\section{Introduction}

A poor response to inhaled $\beta_{2}$ agonists $^{1}$ and delayed response to corticosteroids in a subset of children with acute asthma has brought about interest in the use of mag-

\footnotetext{
Dr Coates and Ms Leung are affiliated with the Physiology and Experimental Medicine Research Institute; Dr Coates is also affiliated with the Division of Respiratory Medicine; Mr Chan, Ms Ribeiro, and Dr Charron are affiliated with the Division of Nuclear Medicine; and Dr Schuh is affiliated with the Department of Emergency Medicine, Hospital for Sick Children, University of Toronto, Toronto, Ontario, Canada.
}

The Idehaler Pocket was supplied at no cost by La Diffusion Technique Francaise. The authors have disclosed no conflicts of interest.

Correspondence: Allan L Coates MDCM, Physiology and Experimental Medicine, Research Institute, Hospital for Sick Children, 555 University Avenue, Toronto, Ontario, Canada M5G1X8. E-mail: allan.coates@ sickkids.ca.

DOI: $10.4187 /$ respcare. 02455 nesium sulfate $\left(\mathrm{MgSO}_{4}\right)$. Two recent meta-analyses ${ }^{2,3}$ suggest a reduction in hospitalization with intravenous $\mathrm{MgSO}_{4}$. However, the intravenous route is associated with a risk of systemic hypotension, which is a major drawback to intravenous $\mathrm{MgSO}_{4}$ in North America. ${ }^{4}$ On the other hand, the aerosolized route of delivery is a noninvasive and direct way to deliver a high concentration of $\mathrm{MgSO}_{4}$ to the airway epithelium and the smooth muscle below without depending on delivery via a high blood level and its associated potential for toxicity. ${ }^{5}$ Although the preliminary evidence suggests clear additive benefit of $\mathrm{MgSO}_{4}$ and albuterol on lung function in adults with severe disease, and a trend toward benefit with respect to lung function and hospitalizations in moderate asthma, ${ }^{2,6}$ the efficacy of nebulized $\mathrm{MgSO}_{4}$ in children is unknown. A major limitation of all studies to date, both in adults ${ }^{7}$ and children, ${ }^{2}$ was the use of inefficient aerosol delivery methods. Given the preliminary evidence of benefit, the noninvasive nature, and the high safety likelihood of the nebulization route, a pediatric study is needed to define the role of 


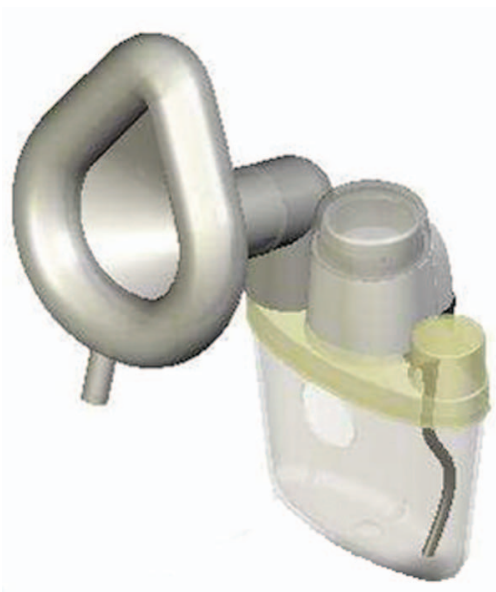

Fig. 1. AeroNeb Go vibrating-mesh nebulizer coupled with the Idehaler Pocket holding chamber, in the configuration used in the in vitro study. ${ }^{10}$ (Courtesy of La Diffusion Technique Francaise.)

nebulized $\mathrm{MgSO}_{4}{ }^{2,6,8}$ Most aerosolized drugs, such as albuterol, are efficacious in the microgram range, whereas $\mathrm{MgSO}_{4}$ is efficacious only in the milligram range and hence requires many-fold higher drug delivery, with a physical limitation of the nebulizable volume rate of output. Increasing the $\mathrm{MgSO}_{4}$ concentration would increase the rate of delivery, but this could be achieved only with aerosol tonicity of $>500 \mathrm{mOsm} / \mathrm{L}$, which has been associated with bronchospasm. ${ }^{9}$

In preparation for a large multicenter study to test the benefit of inhaled $\mathrm{MgSO}_{4}$, in vitro studies were done to select an appropriate delivery system. ${ }^{10}$ Due to both the wide age range of the proposed subjects and the desire to use a single aerosol delivery system, a face mask rather than a mouth piece was required, which precluded the use of high efficiency breath-enhanced or breath-actuated nebulizers, since they require one-way valves and a tight fit with the airway opening. Using breathing patterns ranging from that of adults to small children, in vitro studies suggested that the AeroNeb Go (Aerogen, Galway, Ireland) vibrating-mesh nebulizer, coupled with the novel Idehaler Pocket holding chamber (La Diffusion Technique Francaise, St Etienne, France) (Fig. 1), with a charge volume of $6 \mathrm{~mL}$, would deliver approximately $16 \mathrm{mg} / \mathrm{min}$ of a $100 \mathrm{mg} / \mathrm{mL}$ solution of $\mathrm{MgSO}_{4}$ in older children and adults, and roughly a fifth of this when using a breathing pattern suitable for toddlers. ${ }^{10}$ In that study the in vitro respirable fraction was defined as the mass of aerosol carried in particles $\leq 5 \mu \mathrm{m}$, with the expectation that particles in this size range would, if inhaled, deposit below the vocal cords. Hence, respiratory system deposition was estimated by multiplying the amount of aerosol collected at the filter at the "mouth" by the respirable fraction.

The purpose of this pilot study was to confirm the in vitro estimates in normal adult males, using nuclear medicine

\section{QUICK LOOK}

\section{Current knowledge}

Intravenous $\mathrm{MgSO}_{4}$ may shorten hospitalization in asthma, but $\mathrm{MgSO}_{4}$ is associated with a risk of systemic hypotension. Aerosol is a noninvasive way to deliver a high concentration of $\mathrm{MgSO}_{4}$ directly to the airway epithelium, with a reduced potential for toxicity.

\section{What this paper contributes to our knowledge}

A vibrating mesh nebulizer, coupled with a novel holding chamber, confirmed the deposition predicted from an in vitro model, using a pattern of breathing appropriate for healthy adult males. The tested setup appears to be suitable for a clinical trial of inhaled $\mathrm{MgSO}_{4}$ in children and adults with refractory asthma.

techniques that account for clearance of the aerosol during both the delivery period and any delay between the end of nebulization and imaging. ${ }^{11}$ Confirmation of accuracy for the in vitro data for adults would validate both the performance of this novel delivery system and the in vitro methodology used in the estimates of respiratory system deposition. It is recognized that, ideally, children with acute asthma should be part of the study, but this was considered not acceptable, for ethical reasons.

\section{Methods}

Five healthy non-smoking males between 40 and 67 years of age, who were familiar with the protocol, risks, and methods, participated in the study. Males were chosen to avoid problems with different anterior and posterior chest wall attenuations due to breast tissue. ${ }^{12}$ All participants signed informed consent, and the protocol was approved by our institution's research ethics board. Since there was no reason to suspect that saline would deposit any differently than the aqueous solution $\mathrm{MgSO}_{4}$, normal saline was used in its place, to avoid any pharmacologic effect in the subjects. Based on in vitro data, ${ }^{10}$ approximately $100 \mathrm{MBq}$ of technetium attached to diethylenetriaminepentaacetic acid ( ${ }^{99 \mathrm{~m}}$ Tc-DTPA) was added to $6 \mathrm{~mL}$ of normal saline and placed in the reservoir of the AeroNeb Go, coupled to an Idehaler Pocket holding chamber and a face mask as the patient interface (see Fig. 1). The AeroNeb Go generates a constant flow of aerosol through the membrane, as long as there is fluid on the reservoir side. When there is none, it stops. In order to avoid saturation of the camera from the point source of radiation, ${ }^{12}$ the charged nebulizer was counted on $12 \mathrm{Lu}-$ cite plates $0.5 \mathrm{~cm}$ thick, each with a known attenuation, 
between the nebulizer and the camera. ${ }^{12}$ After acquisition, the counts received by the camera can be "scaled up" (eg, counts divided by 0.4 ) to what they would have been without the Lucite plates. The nebulizer's vibrating mesh is metal and also attenuates the signal, but if the nebulizer is inverted, the thin plastic top has no measurable attenuation (eg, counts in the syringe before loading the nebulizer are the same as counts in the inverted nebulizer after loading). Prior to the inhalation, tissue attenuation was measured using the Macey and Marshal ${ }^{13}$ technique, with a cobalt flood source.

In the aerosol laboratory the subjects inhaled from the device until dryness, which was approximately $12 \mathrm{~min}$. The subject's upper body was in negative-pressure hood evacuated to the outside of the aerosol laboratory to remove any free radioactive aerosol. Following the inhalation, the subject gargled and then drank a glass of water to wash oral and esophageal radiolabel into the stomach. The inhalation was done while seated, but simultaneous anterior and posterior imaging was performed supine to avoid motion artifact. Post-inhalation the subject went to our nuclear medicine facility and was scanned for $1 \mathrm{~min}$, at 5 -min intervals, between the 2 heads of the gamma camera (Millennium MG, GE Healthcare, Madison, Wisconsin), to determine the dose deposited in the lungs, and clearance of the radiolabel from the lungs. Both heads were tuned using a symmetrical $10 \%$ window. Counting was done as soon as practically possible after the end of nebulization, usually within 5-8 min, and then 5, 10, 15, and $20 \mathrm{~min}$ later. Details of this technique have been described previously, ${ }^{11}$ with the exception that it was impossible to compare the amount of radioactivity pre-nebulization to postnebulization, since the absence of an expiratory filter prevented capturing all the aerosol as a measure of quality control.

\section{Calculations}

In order to minimize radiation exposure, a pre-inhalation xenon scan was not done, and the regions of interest were obtained from the first image, when the lungs had the highest number of counts, and which offers the clearest definition of the lung borders ${ }^{14}$ (Fig. 2). Details of the selection of the regions of interest for the stomach, lungs, and mediastinum have been well described recently in healthy subjects. ${ }^{11}$ All counts were corrected for radioactive decay, based on a half-life of 6 hours, starting at the time of imaging of the charged nebulizer pre-nebulization.

During the administration of the aerosol, the ${ }^{99 \mathrm{~m}} \mathrm{Tc}$ DTPA was being constantly removed by absorption into the pulmonary circulation and by mucociliary clearance. Since imaging was carried out at least 15 min after the start of nebulization, the counts in the lung regions at the first acquisition were adjusted for subsequent decline within

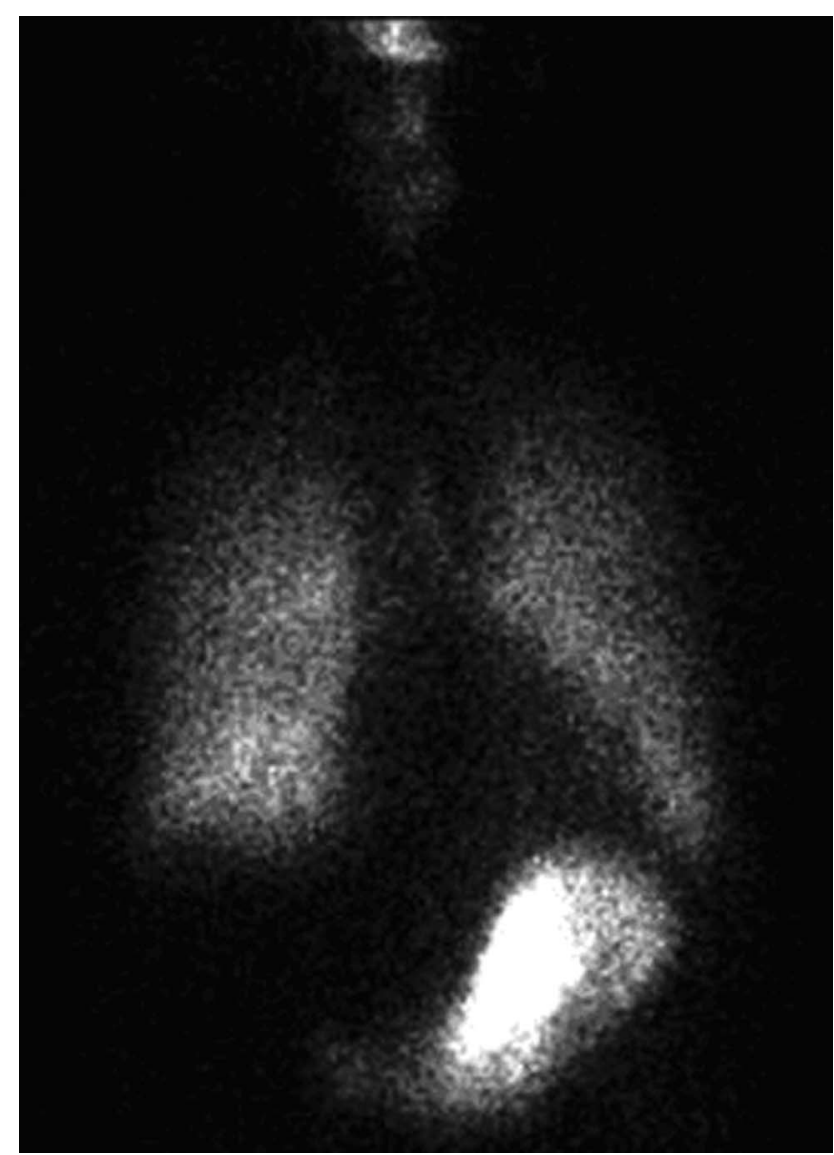

Fig. 2. Example of the first nuclear medicine image after nebulization, illustrating the distinctions between the regions of interest.

that period. The natural logarithm of counts per pixel for the lung regions of interest was plotted against the time of acquisition from the first to the 5th acquisition, occurring 20 min later. ${ }^{11}$ It is assumed that the radiolabel is deposited at a constant rate ${ }^{15}$ and that its clearance is on a time-dependent exponential curve. A linear regression was done using the natural logarithm counts versus time, and the inverse of the slope is the time constant $(\tau)$ of the lung clearance. Linearity of the logarithmic transformed data were assured if the Pearson correlation coefficient was $>0.96$ (which it always was). The total time of nebulization $\left(\mathrm{t}_{\mathrm{f}}\right)$ was then broken into 5 epochs, with the centers of the epochs called $t_{1}, t_{2}, t_{3}, t_{4}$, and $t_{5}$. Hence, for each increment of time, $20 \%$ of the total deposition occurred and part was removed by the lung clearance mechanisms according to the time constant $\tau$. Hence, the amount in the lung at the end of nebulization was:

$$
\begin{aligned}
\Sigma\left[0.2 \mathrm{e}^{-(\mathrm{tf}-\mathrm{t} 1) / \tau)}+0.2 \mathrm{e}^{-(\mathrm{tf}-\mathrm{t} 2) / \tau)}\right. & +0.2 \mathrm{e}^{-(\mathrm{tf}-\mathrm{t} 3) / \tau)} \\
& \left.+0.2 \mathrm{e}^{-(\mathrm{tf}-\mathrm{t} 4) / \tau)}+0.2 \mathrm{e}^{-(\mathrm{ff}-\mathrm{t} 5) / \tau}\right]
\end{aligned}
$$


where $\Sigma$ is the sum and e is the base of the natural logarithm. There is a delay $\left(t_{d}\right)$ between the end of nebulization and the time it takes to move the subject from the aerosol laboratory to the gamma camera and begin counting. Hence the entire expression above was multiplied by $\mathrm{e}^{(-\mathrm{td} / \tau)}$. The resulting value then represents the fraction of the radiation that was deposited in the lung and remained in the lungs at the time of counting. The inverse of this fraction multiplied by the total counts in the first image represented the total pulmonary deposition. Greater details of these calculations have been published recently in both healthy adults ${ }^{11,16,17}$ and children and adults with lung disease. ${ }^{18}$

If $\mathrm{MgSO}_{4}$ had been used instead of saline, the total amount of counts in the nebulizer prior to nebulization would represent $600 \mathrm{mg}$ of $\mathrm{MgSO}_{4}$ in $6 \mathrm{~mL}$ of a $100 \mathrm{mg} / \mathrm{mL}$ solution. Hence, the total counts in the nebulizer prior to nebulization, divided by $600 \mathrm{mg}$, gives the counts per $\mathrm{mg}$ of $\mathrm{MgSO}_{4}$. Hence, the total counts in the lungs and trachea, corrected for all the factors listed above, divided by the total counts in the nebulizer pre-nebulization and multiplied by 600 is the respiratory system deposition expressed as $\mathrm{mg}$ of $\mathrm{MgSO}_{4}$. Dividing by the nebulization time, the rate of deposition is expressed as $\mathrm{mg} / \mathrm{min}$ of $\mathrm{MgSO}_{4}$.

\section{Results}

The subjects ranged in height from 173 to $188 \mathrm{~cm}$, and in weight from 62 to $92 \mathrm{~kg}$. The mean $\pm \mathrm{SD}$ rate of deposition was $12.6 \pm 1.9 \mathrm{mg} / \mathrm{min}$ (95\% CI $10.9-14.2 \mathrm{mg} /$ min, range $11.2-15.9 \mathrm{mg} / \mathrm{min}$ ). Due to both the time for nebulization and the delay in reaching the nuclear medicine department, the mean $\pm \mathrm{SD}$ correction factor for clearance of the radiolabel was $0.87 \pm 0.04$, indicating that $13 \%$ of the lung deposition had been cleared by the time of the first image. The mean $\pm \mathrm{SD}$ in vivo respirable fraction, defined as the lung deposition divided by the total body deposition, was $0.57 \pm 0.05$. Looked at over the course of approximately 12 min of nebulization, roughly $25 \%$ of the charge dose would be deposited in the lungs.

The nebulizations were well tolerated, with no cough or discomfort. Expressing the data in the in vitro study ${ }^{10}$ as the mean and $95 \% \mathrm{CI}$, the $16.0 \mathrm{mg} / \mathrm{min}(95 \%$ CI $15.6-$ $16.5 \mathrm{mg} / \mathrm{min}$ ) does not overlap the $12.6 \mathrm{mg} / \mathrm{min}$ (95\% CI $10.9-14.2 \mathrm{mg} / \mathrm{min}$ ) of the in vivo study, implying significant differences.

\section{Discussion}

This novel nebulizer and holding chamber combination, while not as efficient as modern nebulizing systems ${ }^{17}$ such as the Pari eFlow with one way valves and a tight seal at the airway opening, is certainly as good or better than the breath-enhanced Pari LC Plus nebulizer used for deliver- ing tobramycin. ${ }^{18}$ Roughly $15 \%$ of the $300 \mathrm{mg}$ charge dose in a $5 \mathrm{~mL}$ volume deposited in the lungs in a study including both adults and children, and is roughly the same as a $4 \mathrm{~mL}$ charge delivered by the Pari LC Star in adults. ${ }^{16}$ Importantly, this device is orders of magnitude more efficient than an unvented jet nebulizer with a face mask. ${ }^{19,20}$ Furthermore, while Chua and colleagues ${ }^{19}$ showed extremely low deposition (on the order of $1 \%$ of the emitted nebulizer dose) in infants, they found that roughly $4 \%$ of the emitted dose deposited in the lungs of older children breathing from a face mask, whereas with the novel system in the present study, roughly $25 \%$ of the charge dose deposited in the respiratory system. Hence we would anticipate up to a 5-fold increase if we can extrapolate the difference between their system in older children and the one we used in adults.

The in vivo respirable fraction of $0.57 \pm 0.05$ agreed with that measured via laser diffraction in vitro $(0.59 \pm 0.0),{ }^{10}$ which corresponded to a mass median diameter of $4.4 \pm 0.1 \mu \mathrm{m}$, assuming unit density. The in vitro respirable fraction depends only on measured particle size distribution ${ }^{21}$ and is based on the probability that this fraction of the mass of the particles within a certain size range will, if entering the upper airway, deposit below the vocal cords. Based on previous literature, ${ }^{22-24}$ the in vitro size cutoff was $\leq 5 \mu \mathrm{m}$, and the in vivo agreement is support for this choice. A priori it was recognized that in vivo measurements would be less than in vitro, because during the last phase of inspiration aerosol enters the anatomical dead space, predominately the trachea, and some aerosol that remains in suspension is exhaled. ${ }^{25,26}$ In vivo this does not appear as deposition, but in vitro it is captured on a filter at the "airway opening" of the mechanical breath simulator. During quiet breathing, anatomical dead space represents roughly $25 \%$ of the tidal volume ${ }^{27,28}$ and includes the entire trachea, but tracheal images do indicate deposition, so, while some particles may have been exhaled, others remained.

Despite attempts to wash all the radiolabel out of the upper airway and esophagus, there was a small amount of residual left in the esophagus distal to the bifurcation of the trachea, so it is recognized that including the mediastinum in the deposition below the cords will lead to a slight overestimation of both deposition and the in vivo respirable fraction. However, Figure 2 suggests that esophageal deposition was minimal. It is also recognized that tracheal and main bronchi deposition will most likely have little therapeutic effect on bronchoconstricted airways.

If a constant rate of pulmonary deposition, analogous to an intravenous infusion, is being sought, there are problems with jet nebulizers. First, evaporative loss concentrates drug in the well of the nebulizer, ${ }^{15}$ and hence an increasing rate of aerosol production if measured in quantity of drug per minute over the course of nebulization, 
and increasing osmolarity of the aerosol droplets. Second, toward the end of nebulization sputtering occurs and the production progressively falls. ${ }^{18}$ On the other hand, a vibrating-mesh nebulizer extrudes the drug solution through microscopic pores at a constant rate, with minimal chance for evaporation in the small holding chamber, so the rate of lung deposition and the osmolarity should be constant, providing the pattern of ventilation is constant. ${ }^{11}$ Furthermore, most vibrating-mesh systems have a controller that indicates when nebulization has ceased, allowing the session to be terminated or, alternatively, the nebulizer refilled. This is very similar to constant intravenous infusion via a pump that delivers drug at a constant rate and indicates when the infusion has been completed.

Compared to other studies, where the internal control for accuracy was the agreement of the amount of radioactivity pre-nebulization versus post-nebulization, ${ }^{11,12}$ our study lacked such a control. Since the goal of the study was to test a system with a face mask but without valves, a filter on the expiratory limb was not practical, so aerosol generated that did not deposit in the body was lost to the environment.

A second limitation is the concern as to what degree the results in normal adult males are generalizable to children with acute asthma. While a study on such a population may have been ideal, there were serious ethical concerns about having sick children enrolled in a research study in an area not designed for patients with acute respiratory distress.

While saline was used, rather than $\mathrm{MgSO}_{4}$, there is no reason to suspect there would be differences in the aerosol production or particle size, but it is possible that $\mathrm{MgSO}_{4}$ could change the breathing pattern, either through a response to its taste or tonicity or by changing the pattern of ventilation by reducing bronchospasm in asthmatics. Thus caution is necessary in extrapolating these results into the clinical situation.

The intended comparison in this study was the in vivo deposition in healthy adults to the predicted deposition using an adult pattern of breathing in an in vitro model. ${ }^{10}$ There is no reason to believe that, had it been possible to use small children in this study, there would have been a disagreement with the in vitro model using a suitable pattern of breathing for the age and size range. ${ }^{10}$ Although in vitro data using breath-enhanced nebulizers suggested that, on a $\mathrm{mg} / \mathrm{kg}$ of body weight basis, small children would receive more medication than larger ones, ${ }^{29}$ an in vivo study ${ }^{30}$ in which children over a wide size range inhaled the same dose of tobramycin showed virtually the same blood levels, which strongly implies a drug deposition normalized for size. The most likely explanation for this discrepancy is that the definition of respirable fraction (particles $\leq 5 \mu \mathrm{m}$ ) is not applicable to children with a smaller upper airway. While in vivo data are sparse, mod- eling of the upper airway in infants ${ }^{31}$ would imply that the definition of the respirable fraction should be particles much smaller, perhaps $\leq 3 \mu \mathrm{m},{ }^{32}$ or even smaller. ${ }^{33}$ This would be supported by the in vivo nuclear medicine deposition studies by Chua et al, ${ }^{19}$ who compared deposition from the traditional unvented nebulizer over a large size range in infants and children with cystic fibrosis. Similar conclusions were reached in a deposition study in infants, using metered-dose inhalers with valved holding chambers. ${ }^{34}$ The in vitro data for the delivery system used in that study ${ }^{10}$ did employ varying definitions of respirable fraction and patterns of breathing, based on size of the child, and did suggest that the expected pulmonary dose for the breathing pattern of a small child would be significantly less than that for an older child or adult.

\section{Conclusions}

The AeroNeb Go vibrating-mesh nebulizer, coupled with the Idehaler Pocket holding chamber, did confirm that the deposition predicted from an in vitro model, using a pattern of breathing appropriate for healthy adult males, was within expected limits. However, caution is necessary in extrapolating these results to small children with asthma. The AeroNeb Go appears highly suitable and superior to conventional nebulizers with respect to use in a clinical trial of inhaled $\mathrm{MgSO}_{4}$ in children with refractory asthma. Aerosol delivery would be expected to deposit a relatively high concentration of $\mathrm{MgSO}_{4}$ directly on the respiratory epithelium, which should deliver a higher $\mathrm{MgSO}_{4}$ concentration to the airway smooth muscles than could be safely achieved via intravenous delivery. The AeroNeb Go might facilitate efficacy of inhaled $\mathrm{MgSO}_{4}$ in the treatment of refractory asthma in children, while avoiding adverse effects.

\section{REFERENCES}

1. Fischl MA, Pitchenik A, Gardner LB. An index predicting relapse and need for hospitalization in patients with acute bronchial asthma. N Engl J Med 1981;305(14):783-789.

2. Mohammed S, Goodacre S. Intravenous and nebulised magnesium sulphate for acute asthma: systematc review and meta-analysis. Emerg Med J 2007;24(12):832-830.

3. Cheuk DK, Chau TC, Lee SL. A meta-analysis on intravenous magnesium sulphate for treating acute asthma. Arch Dis Child 2005; 90(1):74-77.

4. Schuh S, Zemek R, Plint A, Black KJL, Freedman S, Porter R, et al. Practice patterns in asthma discharge pharmacotherapy in pediatric emergency departments: a pediatric emergency research Canada study. Acad Emerg Med 2012;19(5):1019-1026.

5. Okayama H, Aikawa T, Okayama M, Saski H, Mue S, Takishima T. Bronchodilating effect of intravenous magnesium sulfate in bronchial asthma. JAMA 1987;257(8):1076-1078.

6. Blitz M, Blitz S, Beasley R, Diner BM, Hughes R, Knopp JA. Inhaled magnesium sulfate in the treatment of acute asthma. Cochrane Database Syst Rev 2005;(4):CD003898. 


\section{Respiratory System Deposition Using a Novel Aerosol Delivery System}

7. Aggarwal P, Sharad S, Handa R, Dwiwedi SN, Irshad M. Comparison of nebulised magnesium sulfate and salbutamol combined with salbutamol alone in the treatment of acute bronchial asthma: a randomised study. Emerg Med J 2006;23(5):358-362.

8. Hughes R, Goldkorn A, Masoli M, Weatherall M, Burgess C, Beasley R. Use of isotonic nebulised magnesium sulfpate as an adjuvant to salbutamol in the treatment of severe asthma: radomised placebo controlled trial. Lancet 2003;361(9375):2114-2117.

9. Dodd M, Abbott J, Maddison J, Moorcroft AJ, Webb AK. Effect of tonicity of nebulized colistin on chest tightness and pulmonary function in adults with cystic fibrosis. Thorax 1997;52(7):656-658.

10. Coates AL, Leung K, Vecellio L, Schuh S. Testing of nebulizers for delivery of magnesium sulfate to pediatric asthma patients in the emergency department. Respir Care 2011;56(3):314-318.

11. Coates AL, Green M, Leung K, Louca E, Tservistas M, Chan J, et al. The challenges of quantitative measurement of lung deposition using ${ }^{99 \mathrm{~m}} \mathrm{Tc}$-DTPA from delivery systems with very different delivery times. J Aerosol Med 2007;20(3):320-330.

12. Coates AL, MacNeish CF, Dinh L, Rollin T, Gagnon S, Ho SL, Lands LC. Accounting for radioactivity before and after nebulization of tobramycin to insure accuracy of quantification of lung deposition. J Aerosol Med 2000;13(3):169-178.

13. Macey DJ, Marshall R. Absolute quantitation of radiotracer uptake in the lungs using a gamma camera. J Nucl Med 1982;23(8):731-735.

14. Brown JS, Zeman KL, Bennett WD. Regional deposition of coarse particles and ventilation distribution in healthy subjects and patients with cystic fibrosis. J Aerosol Med 2001;14(4):443-454.

15. Coates AL, MacNeish CF, Lands LC, Smountas A, Meisner D, Kelemen S, Vadas EB. Factors influencing the rate of drug output during the course of wet nebulization. J Aerosol Med 1998;11(2): 101-111.

16. Coates AL, Green M, Leung K, Chan J, Ribeiro N, Ratjen F, Charron M. A comparison of amount and speed of deposition between the Pari LC Star jet nebulizer and an investigational eFlow nebulizer. J Aerosol Med Pulmon Drug Del 2011;24(3):157-163.

17. Coates AL, Green M, Leung K, Chan J, Ribeiro N, Louca E, et al. Rapid pulmonary delivery of inhaled tobramycin for pseudomonas infection in cystic fibrosis: a pilot project. Pediatr Pulmonol 2008; 43(8):753-759.

18. Coates AL, Denk O, Leung K, Ribeiro N, Chan J, Green M, et al. Higher tobramycin concentration and vibrating mesh technology can shorten antibiotic treatment time in cystic fibrosis. Pediatr Pulmonol 2011;46(4):401-408.

19. Chua HL, Collis GG, Newbury AM, Chan K, Bower GD, Sly PD, LeSouëf PN. The influence of age on aerosol deposition in children with cystic fibrosis. Eur Respir J 1994;7(12):2185-2191.

20. Ilowite JS, Gorvoy JD, Smaldone GC. Quantitative deposition of aerosolized gentamycin in cystic fibrosis. Am Rev Respir Dis 1987; 136(6): 1445-1449.
21. Kwong WTJ, Ho SL, Coates AL. Comparison of nebulized particle size distribution with Malvern laser diffraction analyzer versus Andersen cascade impactor and low-flow Marple personal cascade impactor. J Aerosol Med 2000;13(4):303-314.

22. Coates AL, MacNeish CF, Meisner D, Kelemen S, Thibert R, MacDonald J, Vadas EB. The choice of jet nebulizer, nebulizing flow and the addition of ventolin respiratory solution affects tobramycin aerosols used in cystic fibrosis. Chest 1997;111(5):1206-1212.

23. Newman SP, Pellow PG, Clay MM, Clarke SW. Evaluation of jet nebulisers for use with gentamycin solution. Thorax 1985;40(9): 671-676.

24. Clay MM, Pavia D, Newman SP, Clarke SW. Factors influencing the size distribution of aerosols from jet nebulisers. Thorax 1983;38(10): 755-759.

25. Taulbee DB, Yu CP. A theory of aerosol deposition in the human respiratory tract. J Appl Physiol 1975;38(1):77-85.

26. Brain JD, Valberg PA. Deposition of aerosol in the respiratory tract — state of the Art. Am Rev Respir Dis 1979;120(6):1325-1373.

27. Bates DV, Macklem PT, Christie RV. The normal lung: physiology and methods of study. In: Bates DV, Macklem PT, Christie RV, editors. Respiratory function in disease, 2nd edition. Philadelphia: WB Saunders; 1971:10-95.

28. Chernick V, West JB. The functional basis of respiratory disease. In: Chernick V, Boat TE, Wilmott RW, Bush A, editors. Kendig's disorders of the respiratory tract in children, 7th edition. Philadelphia: Saunders/Elsevier; 2006:29-64.

29. Coates AL, Allen PD, MacNeish CF, Ho SL, Lands LC. Effect of size and disease on expected deposition of drugs given by jet nebulization to children with cystic fibrosis. Chest 2001;119(4): 1123-1130.

30. Geller DE, Pitlick WH, Nardella PA, Tracewell WG, Ramsey BW. Pharmacokinetics and bioavailability of aerosolized tobramycin in cystic fibrosis. Chest 2002;122(1):219-226.

31. Jannsens HM, Krijgsman A, Verbraak TF, Hop WC, de Jongste JC, Tiddens HA. Determining factors of aerosol deposition for four pMDIspacer combinations in an infant upper airway model. J Aerosol Med 2004;17(1):51-61.

32. Coates AL, Tipples GA, Leung K, Gray M, Louca E; WHO Product Development Group for Measles Aerosol Vaccine. How many infective particles are necessary for successful mass measles immunization by aerosol? Vaccine 2006;14(10):1578-1585.

33. Schuepp KG, Jauernig J, Janssens HM, Tiddens HA, Straub DA, Stangl R, et al. In vitro determination of optimal particle size for nebulized aerosol delivery to infants. J Aerosol Med 2005;18(2): 225-235.

34. Tal A, Golan H, Grauer N, Aviram M, Albin D, Quastel MR. Deposition pattern of radiolabeled salbutamol inhaled from a meterdose inhaler by means of a spacer with mask in young children with airway obstruction. J Pediatr 1996;128(4):479-484. 